\title{
Falla hepática aguda en pediatría
}

\author{
Pediatric acute liver failure
}

\section{Angie Milena Cárdenas ${ }^{a}$, Claudia Jimena Ortiz Rivera ${ }^{\mathrm{b}}$, Roberto Andrés Correa ${ }^{\mathrm{c}}$}

aPediatra. Universidad Libre Cali, Miembro del Grupo de Investigación en Pediatría GRINPED COL 0142019, Colombia.

bPediatra gastroenteróloga. Grupo de Investigación en Gastroenterología, Hepatología y Nutrición Pediátrica Gastrohnup. Universidad del Valle. Cali, Colombia.

cPediatra intensivista. Fundación Clínica Infantil Club Noel, Miembro del Grupo de Investigación en Pediatría GRINPED COL 0142019, Colombia.

¿Qué se sabe del tema que trata este estudio?

La FHA en pediatría amenaza la vida, tiene diversas etiologías, requiere un diagnóstico temprano y tratamiento en la unidad de cuidado intensivo pediátrico, el enfoque de pruebas diagnósticas específicas por edad favorece la identificación de la causa.
¿Qué aporta este estudio a lo ya conocido?

Ofrece una revisión de tema con conceptos vigentes acerca de la FHA en pediatría desde la visión del pediatra general, el gastroenterólogo pediatra e intensivista pediatra.

\section{Resumen}

La falla hepática aguda (FHA) es poco frecuente en pediatría, amenaza la vida y requiere abordaje multidisciplinario para su diagnóstico y tratamiento tempranos. El presente artículo tiene como objetivo realizar actualización de definiciones, enfoques y tratamientos disponibles. Los artículos se obtuvieron de la revisión de la literatura disponible entre 2000 y 2020 en varias bases de datos (Pubmed, LILACS, BIREME, Google Académico y UpToDate), empleando términos "acute liver failure" en Pubmed, "pediatric acute liver failure", "falla hepática aguda en pediatría" para otros buscadores y aplicando filtros según edad, fecha de publicación y tipo de estudio (ensayos clínicos, artículos de revisión, revisiones sistemáticas, estudios de casos y controles), se eligieron aquellos artículos con mayor número de citaciones y con datos recientes. La FHA requiere soporte en cuidado intensivo pediátrico y su diagnóstico temprano favorece la instauración del tratamiento. Se recomienda el enfoque de pruebas diagnósticas específicas por edad en pacientes pediátricos con FHA. No hay consenso acerca de las indicaciones de trasplante hepático en FHA en pediatría.

Correspondencia:

Angie Milena Cárdenas

angie-cardenass@unilibre.edu.co 


\begin{abstract}
Acute liver failure (ALF) is a rare and life-threatening entity in pediatrics which requires a multidisciplinary approach for early diagnosis and treatment. The objective of this article is to update definitions, management, and available treatments. We obtained the articles by reviewing the literature available between 2000 and 2020 in different databases (Pubmed, LILACS, BIREME, Google Scholar, and UpToDate), using terms such as "acute liver failure" in Pubmed, and in other databases "pediatric acute liver failure" and "falla hepática aguda en pediatría" using filters such as age, publication date, and types of study (clinical trials, review articles, systematic reviews, and case-control studies). We chose those articles with the highest number of citations and with recent data. The ALF requires support in the pediatric intensive care unit and its early diagnosis favors the beginning of treatment. In pediatric patients with ALF, it is recommended to focus on age-specific diagnostic testing. There is no agreement regarding the liver transplantation in pediatric cases of ALF.
\end{abstract}

\section{Keywords:}

Acute liver failure;

pediatrics;

treatment

\section{Definición}

La FHA ocurre en niños sin hepatopatía previa en un periodo de 8 semanas o menos y se caracteriza por coagulopatía, ictericia y encefalopatía.

El estudio multicéntrico, prospectivo PALF - Pediatric Acute Liver Failure propone los siguientes criterios para definir FHA:

1. Evidencia bioquímica de lesión hepática en ausencia hepatopatía crónica conocida.

2. Coagulopatía de causa hepática que no se corrige con la administración parenteral de fitomenadiona (vitamina K).

3. Relación internacional normalizada (INR) entre 1,5 y 1,9 o su equivalente en tiempo de protrombina (TP) (15 y 19,9 segundos), en paciente con encefalopatía hepática $(\mathrm{EH})$.

4. TP $\geq 20$ segundos o INR $\geq 2$, independiente de la $\mathrm{EH}^{1,2}$.

\section{Epidemiología}

La incidencia de FHA en países desarrollados es 1 a 6 casos por millón de personas año ${ }^{3}$. En pediatría los datos son escasos, aunque se conoce que 10 a $15 \%$ de los trasplantes hepáticos pediátricos se realizan a pacientes con $\mathrm{FHA}^{4}$.

\section{Etiología}

La FHA tiene causas infecciosas, tóxicas, metabólicas, infiltrativas, vasculares y autoinmunes, descritas en la Tabla 1. En los países en vías de desarrollo predomina la etiología infecciosa ${ }^{5}$.

La etiología de la FHA en pediatría se establece en menos del 50\% de los casos ${ }^{1,5}$. En 1999 PALF realizó un estudio en 19 centros asistenciales de Canadá, Reino
Unido y Estados Unidos documentando 548 pacientes. En mayores de 3 años (339 niños) la etiología indeterminada fue $47 \%$, intoxicación por acetaminofén $18 \%$, enfermedad autoinmune $8 \%$, metabolopatías y otras (7\% cada una), medicamentos $6 \%$, virus $4 \%$ e isquemia 4\%. En menores de 3 años (209 casos), la etiología indeterminada fue $54 \%$, metabolopatías $15 \%$, otras causas $12 \%$, virales $8 \%$, isquemia $4 \%$, autoinmunes $3,5 \%$, sobredosis de acetaminofén $3 \%$ y medicamentos diferentes a acetaminofén $0,5 \%{ }^{6}$.

Bitar et al. estudiaron 78 pacientes menores de 120 días de vida e identificaron la etiología en el 94\% de los casos: metabolopatías 36\%, hipóxico-isquémica 19\%, infecciones $17 \%$, hemocromatosis neonatal $9 \%$, enfermedades infiltrativas $9 \%$ e hipopituitarismo $4 \%{ }^{7}$.

\section{Fisiopatología}

Con la inflamación y necrosis hepática hay liberación de material intracelular que resulta en la polimerización de proteínas que impiden el flujo laminar, el intercambio de nutrientes en la microcirculación y alteran la resistencia vascular sistémica lo cual promueve la hipoperfusión e hiperlactatemia.

El aumento de citoquinas proinflamatorias estimula la producción de óxido nítrico $(\mathrm{ON})$ que causa vasodilatación compensatoria. La inflamación y el trastorno en la microcirculación favorecen la hipertensión portal.

El fracaso en la síntesis proteica causa coagulopatía, hipoalbuminemia y disfunción inmunológica. El desbalance entre la respuesta inflamatoria sistémica y la respuesta antiinflamatoria compensatoria predispone a infección. La acumulación de endotoxinas y la hipoperfusión conducen a la injuria y posterior falla renal ${ }^{8}$. Así, la FHA puede llevar a síndrome de disfunción orgánica múltiple. 
Tabla 1. Causas de FHA en pediatría*

\begin{tabular}{|c|c|c|c|c|c|}
\hline Grupo etario & Infecciosas & Intoxicaciones/medicamentos & $\begin{array}{l}\text { Errores innatos del } \\
\text { metabolismo }\end{array}$ & Autoinmunes & Otras \\
\hline $\begin{array}{l}\text { Menores } \\
\text { de un año }\end{array}$ & $\begin{array}{l}\text { Sepsis/shock séptico, } \\
\text { VEB, CMV, VHS, } \\
\text { varicela zoster, } \\
\text { echovirus (Serotipo } \\
\text { 11), VH, enterovirus, } \\
\text { VHH 6, enterovirus, } \\
\text { adenovirus, }\end{array}$ & Acetaminofén & $\begin{array}{l}\text { Galactosemia, } \\
\text { tirosinemia, } \\
\text { fructosemia, } \\
\text { enfermedades } \\
\text { mitocondriales, } \\
\text { trastornos del } \\
\text { ciclo de la urea, } \\
\text { Niemann-Pick tipo C }\end{array}$ & $\begin{array}{l}\text { Hepatitis } \\
\text { autoinmune } \\
\text { Linfohistiocitosis } \\
\text { hemofagocítica, } \\
\text { GALD }\end{array}$ & $\begin{array}{l}\text { Isquemia, } \\
\text { malformaciones }\end{array}$ \\
\hline $\begin{array}{l}\text { Mayores } \\
\text { de un año }\end{array}$ & $\begin{array}{l}\text { Sepsis/shock séptico, } \\
\text { VH, CMV, VEB, } \\
\text { Varicela Influenza, } \\
\text { Adenovirus, } \\
\text { Parvovirus B19 } \\
\text { Tuberculosis }\end{array}$ & $\begin{array}{l}\text { Acetaminofén } \\
\text { Isoniazida } \\
\text { Ácido valproico } \\
\text { AINES } \\
\text { DILI: Trimetropim } \\
\text { sulfametoxazol, tetraciclinas, } \\
\text { macrólidos, aminopenicilinas e } \\
\text { inhibidores de betalactamasas, } \\
\text { sales oro, antitiroideos), } \\
\text { antirretrovirales, ketoconazol, } \\
\text { entre otros } \\
\text { Tóxicas: Amanita phalloides, } \\
\text { ginseng, valeriana, hierba de } \\
\text { San Juan, solventes orgánicos }\end{array}$ & $\begin{array}{l}\text { Enfermedad de } \\
\text { Wilson, trastornos } \\
\text { del ciclo de la urea } \\
\text { y enfermedades } \\
\text { mitocondriales }\end{array}$ & $\begin{array}{l}\text { Hepatitis } \\
\text { autoinmune } \\
\text { Síndrome de } \\
\text { activación } \\
\text { macrofágica }\end{array}$ & $\begin{array}{l}\text { Isquemia, cardiopatías, } \\
\text { Enfermedades } \\
\text { infiltrativas (leucemia y } \\
\text { linfoma) } \\
\text { Síndrome de Reye } \\
\text { Preeclampsia } \\
\text { Anemia de células } \\
\text { falciformes. } \\
\text { Budd-chiari y } \\
\text { enfermedad veno- } \\
\text { oclusiva }\end{array}$ \\
\hline
\end{tabular}

*Modificado de: Reyes-Cerecedo A, et al. ${ }^{17}$. Citomegalovirus CMV; virus de Epstein Barr VEB; virus de herpes simple VHS; virus de hepatitis VH, incluye A, B. C, D y E; VHH virus herpes 6; antiinflamatorios no esteroideos AINES; GALD: gestational alloimmune liver disease - enfermedad hepática aloinmune gestacional. DILI: Drug-induced liver Injury - lesión hepática inducida por medicamentos.

La EH está presente en el 50 a $80 \%$ de los pacientes con FHA severa. En el sistema nervioso central el amonio difunde al interior de la célula y al unirse con glutamato produce glutamina que actúa como osmolito intracelular. La acumulación de amonio incrementa el número de receptores $\mathrm{N}$ metil diaspartato y la producción de ON. El amonio, la glutamina y otros aminoácidos (p. ej. alanina) tienen efecto citotóxico y activan la cascada de inflamación que provoca hiperemia y aumento de la permeabilidad capilar con posterior edema vasogénico. Hay expresión incrementada de acuaporina 4 y cotransportador $\mathrm{Na}+/ \mathrm{K}+/ \mathrm{Cl}$ en los astrocitos que contribuyen al edema celular, pérdida de la autorregulación cerebral por disfunción de la ATPasa $\mathrm{Na}+/ \mathrm{K}+$, elevación de la presión intracraneal, compromiso de la presión de perfusión cerebral e isquemia?

\section{Manifestaciones clínicas}

Las manifestaciones clínicas y bioquímicas se describen en la Tabla 2.

La ictericia es el signo más evidente, aunque los pacientes severamente comprometidos pueden tener hepatitis anictérica.

\begin{tabular}{|c|c|c|}
\hline FHA & & \\
\hline $\begin{array}{l}\text { Alteración en el metabolismo } \\
\text { de carbohidratos, lípidos y } \\
\text { proteínas }\end{array}$ & $\begin{array}{l}\text { Hipoglicemia } \\
\text { Hipoalbuminemia } \\
\text { Coagulopatía } \\
\text { Disfunción inmunológica }\end{array}$ & \\
\hline $\begin{array}{l}\text { Alteración en el aclaramiento } \\
\text { de toxinas y productos finales } \\
\text { del metabolismo. }\end{array}$ & $\begin{array}{l}\text { Ictericia } \\
\text { Hiperamonemia } \\
\text { Acidosis láctica } \\
\text { Encefalopatía } \\
\text { Injuria renal }\end{array}$ & \\
\hline Desbalance SIRS/CARS & $\begin{array}{l}\text { Liberación de citoquinas } \\
\text { proinflamatorias, disminución } \\
\text { de resistencias vasculares } \\
\text { sistémicas, disminución de } \\
\text { volumen circulante efectivo y } \\
\text { formación de tercer espacio, } \\
\text { hipoperfusión, choque }\end{array}$ & $\Sigma$ \\
\hline Disfunción inmunológica & Sepsis & 吕 \\
\hline
\end{tabular}

SIRS: síndrome de respuesta inflamatoria sistémica. CARS síndrome de respuesta antiinflamatoria compensadora. SDOM síndrome de disfunción orgánica múltiple. Fuente: elaboración propia. 
En Turquía, en un estudio con 74 pacientes pediátricos la manifestación clínica inicial más frecuente fue la ictericia $(82,4 \%)$, seguida de hepatomegalia $(63,5 \%)$, esplenomegalia $(29,7 \%)$, ascitis $(21,6 \%)$ y edema pretibial $(21,6 \%)$. Hubo EH en 53/74 pacientes $^{10}$.

En el estudio de Bitar que incluyó niños menores de 120 días de vida las características clínicas fueron ictericia (76\%), hipoglicemia (54\%), falla renal (33\%), sangrado $(28 \%)$ y EH (26\%). Mediante ecografía identificaron ascitis (46\%), hepatomegalia (34\%) y esplenomegalia $(16 \%)^{9}$.

En el estudio de Squires et al. describen EH en $53 \%$ de los pacientes $^{11}$. La evaluación de la $\mathrm{EH}$ en neonatos y lactantes es difícil dado que la irritabilidad o inversión del ciclo sueño vigilia pueden ser las únicas manifestaciones por lo cual la percepción del cuidador de los cambios comportamentales del niño es importante. La Tabla 3 propone la clasificación de la $\mathrm{EH}^{12}$.

\section{Evaluación inicial}

\section{Historia clínica:}

- Cronología de síntomas y signos: ictericia, fiebre, dolor abdominal, náuseas, vómito, aparición de petequias o sangrados, características de la orina y la deposición, cambios comportamentales.

- Uso de medicamentos y medicinas tradicionales.
- Contacto con personas con hepatitis A u otras enfermedades infecciosas.

- Antecedentes familiares de hepatopatías crónicas y metabolopatías

- Condiciones socioeconómicas: residencia urbana o rural, actividad laboral de los padres, hacinamiento, acceso a agua potable y sistemas de alcantarillado, presencia de roedores en la vivienda.

- Historia de transfusiones o consumo de sustancias psicoactivas (cocaína, MDMA - 3,4-metilendioximetanfetamina, Amanita phalloides).

- En neonatos, complicaciones durante el embarazo, tamización TORCHS - toxoplasmosis, rubeola, citomegalovirus, herpes virus y sífilis, antecedentes de mortinatos en la familia o muertes durante el periodo neonatal, consanguinidad de los padres.

- En escolares y adolescentes: evaluar disfunción familiar, acoso escolar, ideación suicida.

- Indagar por inicio de vida sexual y conductas sexuales de riesgo.

\section{Examen físico}

- Signos vitales: taquicardia (choque, infecciones), fiebre (infecciones y autoinmunidad), tensión arterial, glucometría.

- Antropometría: evaluar estado nutricional.

- Signos de enfermedad hepática crónica: ascitis, circulación abdominal colateral, anillos de Kayser Fleischer.

\section{Tabla 3. Encefalopatía hepática en FHA en pediatría}

\begin{tabular}{|c|c|c|c|c|c|}
\hline Estadio & & Clínica & Reflejos & Signos neurológicos & Cambios en el EEG \\
\hline \multirow[t]{2}{*}{ I } & Lactante o niños & $\begin{array}{l}\text { Inconsolable, llanto, poca atención } \\
\text { a las tareas, los padres dicen "no } \\
\text { parece el mismo" }\end{array}$ & $\begin{array}{c}\text { Normal o } \\
\text { hiperreflexia }\end{array}$ & $\begin{array}{l}\text { Difícil o imposible de } \\
\text { evaluar }\end{array}$ & \multirow[t]{2}{*}{$\begin{array}{l}\text { Normal o enlentecimiento } \\
\text { difuso de ritmo theta, } \\
\text { ondas trifásicas }\end{array}$} \\
\hline & Adolescente & $\begin{array}{l}\text { Confusión, cambios en el estado } \\
\text { de ánimo, alteración del ciclo } \\
\text { circadiano, olvidadizo }\end{array}$ & Normal & $\begin{array}{c}\text { Temblor, apraxia, } \\
\text { alteración en la escritura } \\
\text { manual }\end{array}$ & \\
\hline \multirow[t]{2}{*}{$\|$} & Lactante o niños & $\begin{array}{l}\text { Inconsolable, llanto, poca atención } \\
\text { a las tareas, los padres dicen "no } \\
\text { parece el mismo" }\end{array}$ & $\begin{array}{c}\text { Normal o } \\
\text { hiperreflexia }\end{array}$ & $\begin{array}{l}\text { Difícil o imposible de } \\
\text { evaluar }\end{array}$ & \multirow[t]{2}{*}{$\begin{array}{l}\text { Anormal, enlentecimiento } \\
\text { generalizado, ondas } \\
\text { trifásicas }\end{array}$} \\
\hline & Adolescente & $\begin{array}{l}\text { Somnoliento, comportamiento } \\
\text { inapropiado y desinhibido }\end{array}$ & Hiperreflexia & Disartria, ataxia & \\
\hline \multirow[t]{2}{*}{ III } & Lactante o niños & Somnolencia, estupor, combativo & Hiperreflexia & $\begin{array}{l}\text { Difícil o imposible de } \\
\text { evaluar }\end{array}$ & \multirow{2}{*}{$\begin{array}{l}\text { Anormal, enlentecimiento } \\
\text { generalizado, ondas } \\
\text { trifásicas }\end{array}$} \\
\hline & Adolescente & Estupor, obedece órdenes simples & $\begin{array}{l}\text { Hiperreflexia, } \\
\text { Babinski + }\end{array}$ & Rigidez & \\
\hline \multirow[t]{2}{*}{ IV } & Lactante o niños & $\begin{array}{l}\text { Coma, alertable con estímulos } \\
\text { dolorosos (IVa) o no responde } \\
\text { (IVb) }\end{array}$ & Ausente & $\begin{array}{l}\text { Decerebración o } \\
\text { decorticación }\end{array}$ & \multirow[t]{2}{*}{$\begin{array}{l}\text { Anormal, ondas muy } \\
\text { lentas, actividad delta }\end{array}$} \\
\hline & Adolescente & $\begin{array}{l}\text { Coma, alertable con estímulos } \\
\text { dolorosos (IVa) o no responde }\end{array}$ & Ausente & $\begin{array}{l}\text { Decerebración o } \\
\text { decorticación }\end{array}$ & \\
\hline
\end{tabular}

Modificado de 12. 
- Cardiovascular: precordio hiperdinámico, taquicardia o bradicardia, hipotensión o hipertensión, llenado capilar.

- Respiratorio: bradipnea, taquipnea, polipnea, ruidos agregados pulmonares.

- Gastrointestinal: tolerancia a la vía oral, sangrado digestivo, acolia, vomito, dolor abdominal, hepatomegalia (la disminución abrupta del tamaño del hígado es signo de gravedad ${ }^{13}$, esplenomegalia.

- Renal: edemas, estertores o crépitos, diuresis, coluria o hematuria.

- Neurológico: neurodesarrollo previo. Interrogar por alteraciones en el comportamiento reconocidos por el cuidador, conexión con el medio, convulsiones, irritabilidad, inversión del ciclo circadiano, asterixis.

- Esfera mental: ideación suicida, depresión.

- Piel y anexos: ictericia y evidencia de sangrado. Signos de autoagresión.

\section{Laboratorio}

Los exámenes apoyan el diagnóstico clínico y evalúan la respuesta al tratamiento y evolución. Los estudios iniciales permiten valorar la lesión hepática y la repercusión en otros órganos y sistemas, como se describen a continuación:

- Lesión del hepatocito: alanino aminotransferasa (ALT), aspartato aminotransferasa (AST), bilirrubinas, gamma glutamil transferasa, fosfatasa alcalina.

ALT se encuentra en el hígado principalmente, mientras AST está en el hígado, músculo cardiaco, riñón, cerebro, páncreas, pulmones, leucocitos y eritrocitos por lo cual es menos específica para enfermedad hepática ${ }^{14}$. La disminución abrupta de aminotransferasas refleja necrosis hepatocelular masiva y es indicador de mal pronóstico en FHA ${ }^{1}$.

- Alteración en el metabolismo de carbohidratos, lípidos y proteínas: glucosa en suero, albúmina, fibrinógeno, TPT, TP e INR, estos últimos no son buenos predictores de riesgo de sangrado debido a que en FHA hay disminución de la síntesis de factores procoagulantes y anticoagulantes.

- Alteración en el aclaramiento de toxinas y productos finales del metabolismo: bilirrubinas, amonio en sangre, gasometría y lactato, uroanálisis, nitrógeno ureico, creatinina, electrolitos séricos.

- Disfunción inmunológica: hemograma, hemocultivos.

Narkewicz et al. proponen el enfoque de pruebas diagnósticas específicas por edad, en su estudio la implementación de esta estrategia disminuyó hasta 30,8\% la etiología indeterminada en FHA y sugiere que podría reducir la necesidad de trasplante hepático $(\mathrm{TH})$ sin incrementar la mortalidad ${ }^{15}$. En la Tabla 4 se describen las pruebas diagnósticas específicas por edad.

Imágenes: La ecografía abdominal y hepática con Doppler permite evaluar la permeabilidad del flujo sanguíneo hepático (Ej. síndrome de Budd Chiari). La radiografía de tórax para descartar consolidaciones, hiperflujo pulmonar o derrame pleural.

Biopsia hepática: El papel de la biopsia hepática en FHA es limitado y cuestionado ${ }^{16}$. Reyes-Cerecedo et al. la recomiendan en el estudio de síndrome colestásico, hipertensión portal, hepatomegalia no explicada, sospecha de metabolopatías y lesiones infiltrativas, fiebre de origen desconocido y evaluación del grado de fibrosis y posibilidad de $\mathrm{TH}^{17}$. La vía transyugular está indicada en pacientes con coagulopatía severa, ascitis, obesidad masiva y lesión vascular ${ }^{18}$.

\section{Tratamiento}

El paciente pediátrico con FHA requiere manejo por un equipo multidisciplinario en cuidado intensivo. El tratamiento está dirigido a:

I. Buscar la causa FHA.

II. Monitorizar la función de cada órgano.

III. Identificar y tratar complicaciones.

IV. Proveer soporte para maximizar la salud y la sobrevivencia ${ }^{5}$.

\section{Buscar la causa de FHA}

Con base en la historia clínica y el examen físico, se sugiere realizar el enfoque de pruebas diagnósticas específicas por edad. La identificación de la causa favorece la instauración del manejo dirigido (Tabla 5).

\section{Monitorizar la función de cada órgano}

Vigilancia de la evolución de acuerdo con parámetros clínicos y de laboratorio, ajustando las intervenciones a las necesidades y contexto del paciente.

\section{Identificar y tratar complicaciones}

Hipoglicemia. Secundaria a la alteración en la gluconeogénesis hepática y la depleción de las reservas de glucógeno. Favorecer aportes de glucosa suficientes para mantener glicemia entre 90 y $110 \mathrm{mg} / \mathrm{dl}^{19}$.

Coagulopatía. La FHA lleva a disfunción plaquetaria, hipofibrinogenemia y déficit de vitamina K. Los tiempos de coagulación se corrigen en presencia de sangrado activo o necesidad de procedimiento quirúrgico. Se requiere vitamina $\mathrm{K}$ intravenosa como profilaxis $^{20}$.

Encefalopatía hepática. Vigilar signos de EH mientras se mantienen medidas antiedema: cabecera a 30 grados, $\mathrm{SaO} 2>95 \%, \mathrm{PaCO} 2$ entre 30 y 35 mm Hg${ }^{19}$. Ante la sospecha de edema cerebral se puede usar solu- 
Tabla 4. Recomendaciones para pruebas diagnósticas específicas por edad en FHA en pediatría

\begin{tabular}{|c|c|c|c|c|c|}
\hline \multirow[t]{2}{*}{ Prueba recomendada } & \multirow[t]{2}{*}{ Indicación } & \multicolumn{4}{|c|}{ Pruebas diagnósticas específicas por edad } \\
\hline & & $\begin{array}{l}<3 \\
\text { meses }\end{array}$ & $\begin{array}{l}3 \text { meses } \\
\text { a } 3 \text { años }\end{array}$ & $\begin{array}{l}3 \text { meses } \\
\text { a } 18 \text { años }\end{array}$ & $\begin{array}{l}4-18 \\
\text { años }\end{array}$ \\
\hline \multicolumn{6}{|l|}{ Estudios en sangre y en orina } \\
\hline \multicolumn{6}{|l|}{ Enfermedades infecciosas } \\
\hline PCR para herpes en sangre & Infección sistémica por herpes & $x$ & & $x$ & \\
\hline PCR para enterovirus en sangre & Infección sistémica por enterovirus & $x$ & $x$ & & \\
\hline IgM para hepatitis A & Infección por hepatitis A & & & $x$ & \\
\hline HBsAg & & & & $x$ & \\
\hline$P C R$ o IgM ACV VEB & & & & $x$ & \\
\hline Confirmar la serología materna para hepatitis B & Hepatitis B en el neonato & $x$ & & & \\
\hline \multicolumn{6}{|l|}{ Enfermedades metabólicas } \\
\hline Perfil de aminoácidos séricos & $\begin{array}{l}\text { Trastornos del ciclo de la urea u otros } \\
\text { trastornos metabólicos }\end{array}$ & $x$ & & $x$ & \\
\hline Lactato, piruvato & Tamizaje mitocondrial & $x$ & & $x$ & \\
\hline Perfil de acilcarnitina plasmático & Trastornos de la oxidación de los ácidos grasos & $x$ & & $x$ & \\
\hline Succinilacetona urinario & Tirosinemia & $x$ & & & \\
\hline Ceruloplasmina & Enfermedad de Wilson & & & & $x$ \\
\hline Cobre en orina de 24 horas & Enfermedad de Wilson & & & & $x$ \\
\hline Actividad enzimática GALT & Galactosemia & $x$ & & & \\
\hline \multicolumn{6}{|l|}{ Toxicología } \\
\hline Niveles de acetaminofén & Exposición a acetaminofén & & & $x$ & \\
\hline \multicolumn{6}{|l|}{ Enfermedades autoinmunes } \\
\hline ANAS & Enfermedades autoinmunes & & & $x$ & \\
\hline Ac anti músculo liso & Enfermedades autoinmunes & & & $x$ & \\
\hline Ac microsomales de hígado riñón & Enfermedades autoinmunes & & & $x$ & \\
\hline $\lg G$ & Enfermedades autoinmunes & & & $x$ & \\
\hline Ferritina & Tamizaje para GALD & $x$ & & & \\
\hline \multicolumn{6}{|l|}{ Estudios de imagen } \\
\hline Ecografía abdominal con Doppler & Alteraciones vasculares & $x$ & & $x$ & \\
\hline Ecocardiograma & Disfunción cardiaca & $x$ & & $x$ & \\
\hline
\end{tabular}

Modificado de ref 15. Ac: anticuerpos; ACV: antígenos capsulares virales; ANAS anticuerpos antinucleares; HBsAg: Antígeno de superficie para hepatitis B. IgM inmunoglobulina M; IgG: Inmunoglobulina G; GALD, gestational alloimmune liver disease - enfermedad hepática autoinmune gestacional; GALT: galactosa-1-fosfato uridiltransferasa PCR: reacción en cadena de la polimerasa; VEB: virus Epstein-Barr.

ción salina hipertónica 3\%, manteniendo sodio sérico entre 145 y $150 \mathrm{mEq} / \mathrm{l}^{21}$.

La lactulosa remueve las sustancias que contienen nitrógeno del tracto gastrointestinal por sus efectos laxantes y es metabolizada por la microbiota colónica para producir ácidos orgánicos de cadena corta que inhiben el crecimiento de bacterias que producen amonio $^{22}$. La dosis recomendada es $0,5 \mathrm{ml} / \mathrm{kg} /$ dosis (hasta $30 \mathrm{ml}$ dosis) y ajustarla hasta lograr entre 2 y 4 deposiciones diarias ${ }^{23}$.

La rifaximina (antibiótico de acción luminal) reduce los efectos de la flora intestinal, incluyendo especies productoras de amonio ${ }^{22}$. Su eficacia es similar a lactulosa en los adultos, pero los datos en niños son escasos $^{12}$.
Evitar la sedación y de ser necesaria no usar benzodiacepinas, se prefiere propofol (en periodos cortos) u opioides.

Disfunción cardiovascular. En pacientes en estado de choque el tratamiento es mantener volumen intravascular adecuado y usar vasopresores en quienes no responden a la reanimación hídrica² ${ }^{2}$.

Insuficiencia suprarrenal relativa. El uso de esteroides puede considerarse en choque refractario a catecolaminas ${ }^{24}$.

Síndrome de dificultad respiratoria aguda (SDRA). La incidencia SDRA en pacientes pediátricos con FHA es desconocida. El manejo de SDRA se realiza según las recomendaciones de PALICC- Pediatric Acute Lung Injury Consensus Conference, manteniendo la nor- 
Tabla 5. Causas de falla hepática con tratamiento específico

\begin{tabular}{|c|c|c|}
\hline Etiología & Tratamiento & Observaciones \\
\hline \multicolumn{3}{|l|}{ Tóxicas } \\
\hline $\begin{array}{l}\text { Intoxicación por } \\
\text { acetaminofén }\end{array}$ & $\begin{array}{l}\text { NAC. Bolo inicial: } 50-150 \mathrm{mg} / \mathrm{kg} \\
\text { Tiempo de infusión: } 15-60 \mathrm{~min} \text {, } \\
\text { seguido de infusión intermitente } \\
\text { o continua en las próximas } 20-48 \mathrm{~h}\end{array}$ & $\begin{array}{l}\text { NAC no debe usarse en FHA secundaria a otras causas diferentes a } \\
\text { intoxicación por acetaminofén. En el estudio doble ciego, controlado } \\
\text { de Squires et al. comparan el uso de NAC vs placebo, encontrando } \\
\text { que NAC no impacta en la sobrevida y el pronóstico a un año sin } \\
\text { trasplante hepático fue menor en pacientes tratados con NAC }{ }^{32}\end{array}$ \\
\hline $\begin{array}{l}\text { Intoxicación por Amanita } \\
\text { phalloides }\end{array}$ & $\begin{array}{l}\text { Carbón activado } \\
\text { Silibinina IV } \\
\text { Manejo de soporte }^{33}\end{array}$ & \\
\hline \multicolumn{3}{|l|}{ Metabólicas } \\
\hline Enfermedad de Wilson & $\begin{array}{l}\text { Quelación del cobre (tienteno o } \\
\text { penicilamina) y zinc } \\
\text { Plasmaféresis }\end{array}$ & \\
\hline Tirosinemia tipo 1 & Nitisinona (NTBC), dieta baja en tirosina & \\
\hline Galactosemia & Fórmula libre de lactosa & \\
\hline \multicolumn{3}{|l|}{ Infecciosas } \\
\hline Hepatitis B & Entecavir o tenofovir & $\begin{array}{l}\text { Los recién nacidos de madres con VHB+ deben recibir vacuna e } \\
\text { inmunoglobulina para hepatitis B }\end{array}$ \\
\hline Virus del herpes simple & Aciclovir & \\
\hline \multicolumn{3}{|l|}{ Autoinmunes } \\
\hline Hepatitis autoinmune & Metilprednisolona IV en bolo & \\
\hline GALD & Exanguinotransfusión e lgG IV & \\
\hline $\begin{array}{l}\text { Linfohistiocitosis } \\
\text { hemofagocítica }\end{array}$ & $\begin{array}{l}\text { Inmunosupresión, trasplante de médula } \\
\text { ósea o células madre }\end{array}$ & \\
\hline \multicolumn{3}{|l|}{ Vasculares } \\
\hline Síndrome de Budd Chiari & $\begin{array}{l}\text { Derivación porto sistémica transyugular } \\
\text { intrahepática (TIPS) }\end{array}$ & \\
\hline
\end{tabular}

NAC: N acetil cisteína TIPS: transjugular intrahepatic portosystemic shunt. Modificado de la referencias 2 y 12.

mocapnia y evitando la hipoxemia. Permitir la hiperventilación ante signos de elevación súbita de la presión intracraneal, pero la hiperventilación mantenida se desaconseja ${ }^{2}$.

Insuficiencia renal. Las etiologías de falla renal aguda en FHA en pediatría incluyen: intoxicación por acetaminofén, medicamentos nefrotóxicos, infección o hipovolemia. De ser necesaria, se prefiere la terapia de reemplazo renal continua ${ }^{2}$.

\section{Proveer soporte para maximizar la salud y la sobrevivencia}

Temperatura. Se recomienda la normotermia (36$37^{\circ} \mathrm{C}$ ) considerando la relación riesgo beneficio ${ }^{2}$.

Nutrición. Se prefiere la nutrición enteral con aportes calóricos de $150 \%$, carbohidratos $15-20 \mathrm{~g} / \mathrm{kg} /$ día, grasas $8 \mathrm{~g} / \mathrm{kg} /$ día con $50 \%$ como triglicéridos de cadena media ${ }^{19}$ y proteína $1 \mathrm{~g} / \mathrm{kg} /$ día (disminuir a $0,5 \mathrm{~g} / \mathrm{kg} /$ día en presencia de hiperamonemia o EH) y evitar admi- nistración de aminoácidos de cadena ramificada ${ }^{25}$.

Profilaxis para hemorragia de vías digestivas. Aunque el sangrado digestivo es raro en pacientes pediátricos con $\mathrm{FHA}^{2}$, todos ellos deben recibir inhibidores de bomba de protones como profilaxis primaria.

Manejo de líquidos y electrolitos. Se recomienda restricción hídrica entre 90 y 95\% de los requerimientos basales ${ }^{12}$ y monitorización de electrolitos séricos y corrección temprana de sus alteraciones.

Infecciones. Realizar búsqueda activa de posibles focos infecciosos; la profilaxis antibiótica se desaconseja $\mathrm{a}^{20}$.

\section{Trasplante hepático}

Aunque no hay consenso en pediatría para definir la necesidad de TH los criterios de King's College son los más aceptados y consideran dos grupos: 
1. FHA secundaria a intoxicación por acetaminofén en cuyo caso $\mathrm{pH}<7,3$ o INR $>6,5$, creatinina sérica $>3,4 \mathrm{mg} / \mathrm{dl}$ y EH III/IV son criterios de TH.

2. FHA por otras etiologías: INR $>6,5$ o tres de los siguientes criterios: edad $<10$ o $>40$ años, hepatitis no A y no B o reacciones idiosincráticas a medicamentos, instauración de la ictericia $>7$ días previo a la $\mathrm{EH}, \mathrm{INR}>3,4$ y bilirrubina sérica $>17,5 \mathrm{mg} / \mathrm{dl}$ son criterios para $\mathrm{TH}^{26}$.

Decidir el momento para incluir a un paciente en la lista de $\mathrm{TH}$ es un reto, hacerlo muy pronto significa realizar un procedimiento quirúrgico invasivo e inmunosuprimir a quien pudo recuperarse con terapia de soporte, mientras retrasar su inclusión representa riesgo de deterioro clínico y desarrollo de comorbilidades hasta que el paciente esté "demasiado enfermo" para recibir un TH. Squires JE et al. realizaron un estudio con datos del registro PALF entre 1999 y 2014, encontraron que 385 pacientes fueron incluidos en la lista de $\mathrm{TH}$, predominó el sexo masculino (58\%), etiología indeterminada en 59\%, INR elevado (promedio $3 \mathrm{~s}$ ), bilirrubinas totales elevadas (promedio $15 \mathrm{mg} / \mathrm{dl}$ ), hiperlactatemia (promedio 2,8) e hiperamonemia (promedio $63 \mu \mathrm{mol} / \mathrm{L}), 26 \%$ tenían ventilación mecánica y $12 \%$ soporte vasoactivo ${ }^{27}$.

Todo paciente con FHA debe ser derivado oportunamente a un centro de trasplante hepático, especialmente en caso de rápido ascenso de INR o evidencia de encefalopatía hepática.

\section{Soporte hepático extracorpóreo}

Entre 15 y $22 \%$ de los pacientes con FHA fallecen mientras esperan un trasplante hepático de emergen$\mathrm{cia}^{28}$, los sistemas de soporte hepático extracorpóreo son una alternativa incipiente en cuidado intensivo pediátrico.

La terapia MARS (Molecular Adsorbent Recirculating System) consiste en un circuito primario por el que fluye la sangre del paciente a través de un filtro (de menos de $60 \mathrm{KDa}$ ) y un circuito secundario con un dializado rico en albumina en contracorriente. Las sustancias unidas a albúmina en el primer circuito se disocian, atraviesan el filtro y se adhieren a la albúmina del segundo circuito, se dializan para remover partículas hidrosolubles y la albúmina del dializante se reactiva por adsorción con carbón activado y una resina aniónica ${ }^{29}$.

En pediatría los datos son escasos. Shaefer et al. comparan pacientes tratados con MARS vs plasmaféresis y hemodiálisis, encontrando mejoría de la coagulopatía y mayor eficacia en la remoción de toxinas hidrosolubles y unidas a albumina en el último grupo ${ }^{30}$. Bernabéu y su grupo emplearon MARS en pacientes con EH II o mayor, insuficiencia hepatorrenal o intoxicación medicamentosa susceptible de ser aclarada con este sistema, hubo disminución en la concentración de toxinas hidrosolubles y unidas a proteínas ${ }^{31}$.

\section{Conclusiones}

- La FHA es una condición clínica que amenaza la vida, secundaria a múltiples etiologías, requiere soporte en cuidado intensivo pediátrico, su diagnóstico temprano favorece la instauración del tratamiento.

- Se recomienda el enfoque de pruebas diagnósticas específicas por edad en pacientes pediátricos con FHA.

- La identificación de la causa de FHA favorece la instauración del tratamiento específico.

- No hay consenso acerca de las indicaciones de trasplante hepático en FHA en pediatría.

- La terapia MARS es una alternativa en el tratamiento de pacientes con FHA severa, aunque se requieren estudios adicionales en pediatría para recomendar su uso rutinario.

\section{Conflicto de intereses}

Los autores declaran no tener conflicto de intereses.

\section{Referencias}

1. Squires R. Acute Liver Failure in Children. Semin Liver Dis. 2008;28:153-66. DOI 10.1055/s-2008-1073115.

2. Lutfi R, Abulebda K, Nitu ME, Molleston JP, Bozic MA, Subbarao G. Intensive Care Management of Pediatric Acute Liver Failure. J Pediatr Gastroenterol Nutr. 2017;64(5):660-70. DOI: 10.1097/ MPG.0000000000001441.
3. Montrief T, Koyfman A, Long B. Acute liver failure: A review for emergency physicians. American Journal of Emergency Medicine. 2019;(37):329-37. Disponible en: https://doi.org/10.1016/j. ajem.2018.10.032.

4. Newland CD. Acute Liver Failure. Pediatr Ann. 2016;45(12):e433-e438. DOI: 10.3928/19382359-20161128-01.

5. Squires RH, Alonso EM. Acute liver failure in children. In: Liver Disease in Children, 4th ed, Suchy FJ, Sokol RJ, Balistreri WF (Eds), Cambridge University Press, New York 2012. Copyright $\odot 2012$ Cambridge University Press.

6. Lee W, Squires R, Nyberg S, Doo E, Hoofnagle J. Acute Liver Failure: Summary of a Workshop. Hepatology. 2008; 47(4):1401-15. DOI: 10.1002/ hep.22177.

7. Bitar R, Thwaites R, Davison S, 
Rajwal S, McClean P. Liver failure in early infancy: Aetiology, presentation, and outcome. J Pediatr Gastroenterol Nutr. 2017;64(1):70-5. DOI: 10.1097/ MPG.0000000000001202.

8. Larsen FS, Bjerring PN. Acute liver failure. Curr Opin Crit Care. 2011;17(2):160-4.

9. Mendes P, Nogueira R, Oliveira M, et al. Cerebral hemodynamic and metabolic changes in fulminant hepatic failure. Arq. Neuro-Psiquiatr. 2017;75(7):4706. Disponible en: http://dx.doi. org/10.1590/0004-282x20170076.

10. Baris Z, Temizel S, Uslu N, et al. Acute liver failure in children: 20year experience. Turk J Gastroenterol 2012;23(2):127-34. DOI: $10.4318 /$ tjg.2012.0319.

11. Squires RH, Shneider BL, Bucuvalas $\mathrm{J}$, et al. Acute liver failure in children: The first 348 patients in the pediatric acute liver failure study group. J Pediatr. 2006;148(5):652-8.

12. Squires J, McKiernan P, Squires R. Acute Liver Failure. An Update. Clin Liver Dis. 2018; (22):773-805. DOI: https://doi. org/10.1016/j.cld.2018.06.009.

13. Devictor D, Tissieres P, Afanetti M, Debray D. Acute liver failure in children. Clin Res Hepatol Gastroenterol. 2011;35(6-7):430-7. DOI: 10.1016/j.clinre.2011.03.005.

14. Pratt D. Bioquímica y pruebas de función hepáticas. En: Enfermedades digestivas y hepáticas. Sleisenger y Fordtran. 10 Ed. Capítulo 73. Elsevier, España 2018. p. 1245.

15. Narkewicz M, Horslen S, Hardison R, et al. A Learning Collaborative Approach Increases Specificity of Diagnosis of Acute Liver Failure in Pediatric Patients. Clin Gastroenterol Hepatol. 2018;16(11):180110.e3. DOI: $10.1016 /$ j.cgh.2018.04.050.

16. Dezsőfi A, Baumann U, Dhawan A, et al. Liver biopsy in children: position paper of the ESPGHAN Hepatology Committee. J Pediatr Gastroenterol Nutr. 2015;60(3):408-20. Disponible en: http:// europepmc.org/abstract/MED/25383787. DOI: 10.1097/MPG.0000000000000632.
17. Reyes-Cerecedo A, Flores-Calderón J, Villasis-Keever MA, et al. Consenso para el manejo de la falla hepática aguda en pediatría. Rev Mex Pediatr 2017;84(3):120-8.

18. Almeida P, Schreiber RA, Liang J, Mujawar Q, Guttman OR. Clinical Characteristics and Complications of Pediatric Liver Biopsy: A Single Centre Experience. Ann Hepatol. 2017;16(5):797801. DOI: $10.5604 / 01.3001 .0010 .2809$.

19. Huang J, Rudnick DA. Elucidating the metabolic regulation of liver regeneration. Am J Pathol 2014;184:309.

20. Ciocca M, Costaguta A, Cuarterolo M, Delgado L, Garcete L, Godoy M, et. al. Insuficiencia hepática aguda pediátrica. grupo de trabajo de la sociedad latinoamericana de gastroenterología, hepatología y nutrición pediátrica (SLAGHNP). Acta Gastroenterol Latinoam 2016;46(1):52-70.

21. Tissieres P, Devictor DJ. Acute Liver Failure and Liver Transplantation. En: Shaffner D; Nichols D. Rogers' Textbook of Pediatric Intensive Care. Wolters Kluwer. 2015. Pág 1706-25.

22. Hudson M, Schuchmann M. Long-term management of hepatic encephalopathy with lactulose and/or rifaximin: a review of the evidence. Eur J Gastroenterol Hepatol. 2019;31(4):434-50. Disponible en: https://www.ncbi.nlm.nih.gov/ pubmed/30444745. DOI: 10.1097/ MEG.0000000000001311.

23. Debray D, Yousef N, Durand P. New management options for end-stage chronic liver disease and acute liver failure: potential for pediatric patients. Paediatr Drugs 2006;8:1

24. Weiss SL, Peters MJ, Alhazzani W, et al. Surviving sepsis campaign international guidelines for the management of septic shock and sepsis-associated organ dysfunction in children. Intensive Care Med. 2020;46(Suppl 1):10-67. Disponible en: http://www.ncbi.nlm.nih.gov/ pubmed/32030529.

25. Dam G, Keiding S, Munk OL, et al. Branched-chain amino acids increase arterial blood ammonia in spite of enhanced intrinsic muscle ammonia metabolism in patients with cirrhosis and healthy subjects. Am J Physiol Gastrointest Liver Physiol 2011;301: G269.

26. Castaldo ET, Chari RS. Liver transplantation for acute hepatic failure. HPB (Oxford) 2006;8(1):29-34. DOI: $10.1080 / 13651820500465741$.

27. Squires JE, Rudnick DA, Hardison RM, et al. Liver Transplant Listing in Pediatric Acute Liver Failure: Practices and Participant Characteristics. Hepatology. 2018;68(6):2338-47. DOI:10.1002/ hep.30116.

28. Lexmond WS, Van Dael CML, Scheenstra R, et al. Experience with molecular adsorbent recirculating system treatment in 20 children listed for high-urgency liver transplantation. Liver Transplant. 2015;21(3):369-80. DOI: $10.1002 / 1$ t.24037.

29. Jain V, Dhawan A. Extracorporeal Liver Support Systems in Paediatric Liver Failure. J Pediatr Gastroenterol Nutr. 2017;64(6):855-863. DOI: 10.1097/ MPG.0000000000001500.

30. Schaefer B, Schaefer F, Engelmann G, Meyburg J, Heckert KH, Zorn M, Schmitt C P. Comparison of Molecular Adsorbents Recirculating System (MARS) dialysis with combined plasma exchange and haemodialysis in children with acute liver failure. Nephrol Dial Transplant. 2011;26:3633-39. DOI: $10.1093 / \mathrm{ndt} /$ gfr115.

31. Quintero Bernabeu J, Ortega López J, Juamperez Goni J, et al. The role of molecular adsorbent recirculating system in pediatric acute liver failure. Liver Transpl. 2018;24(2):308-310. DOI: 10.1002/lt.24966.

32. Squires RH, Dhawan A, Alonso E, et al. Intravenous $\mathrm{N}$-acetylcysteine in pediatric patients with nonacetaminophen acute liver failure: A placebo-controlled clinical trial. Hepatology. 2013;57(4):1542-9.

33. Goldfrank LR. Mushrooms. En: Goldfrank's Toxicologic Emergencies, $9^{\text {th }}$, Nelson LS, Lewin NA, Howland MA, et al. McGraw-Hill, New York 2011. p.1522. 